\title{
Mid-Infrared Octave Spanning Frequency Comb Generation in Silicon Microrings
}

\author{
T. Hansson ${ }^{(1)}$, D. Modotto ${ }^{(1)}$, S. Wabnitz ${ }^{(1)}$
}

(1) Dipartimento di Ingegneria dell'Informazione, Università di Brescia, Brescia, Italy tobias.hansson@unibs.it; daniele.modotto@unibs.it; stefan.wabnitz@unibs.it

\begin{abstract}
We numerically show that octave-spanning Kerr frequency comb generation in the MIR spectral region may be obtained from planar microring resonators, by combining analytical dispersion engineering with generalized nonlinear envelope equation simulations of the temporal frequency comb dynamics.
\end{abstract}

\section{Introduction}

Optical frequency comb generation using microresonators is currently an active field of research. Microresonator based Kerr frequency combs offer an intriguing alternative to modelocked lasers, with a host of potential applications including, e.g., spectroscopy and precision frequency metrology ${ }^{1-2}$. Planar microring resonators that can be fabricated directly on CMOS compatible chips are particularly interesting due to their low cost and potential for mass manufacturing. Moreover, there is a strong interest to develop comb sources in the mid-infrared (MIR) range of the spectrum, which is vital for biomedical and environmental applications. The natural candidate for implementing a chip-scale, MIR Kerr frequency comb source is silicon, since two-photon absorption (TPA) and free-carrier absorption (FCA) vanish for photon energies below the silicon half band-gap.

In this work we present a theoretical study of octave-spanning, silicon-based MIR frequency comb sources. Field evolution inside the miicroresonators is fundamentally described by the vector wave equation for the electric field inside the cavity. The wave equation can, under suitable approximations, be separated into a spatial part for the mode profiles and the eigenfrequencies of the resonant modes, as well as a temporal part which describes the dynamical evolution of the mode amplitudes. Accurate modeling of microresonator frequency combs requires both the solution of the spatial problem for the determination of the cavity eigenmodes and eigenfrequencies, as well as the solution of the temporal problem for describing the evolution of the mode amplitudes and the associated nonlinear dynamics responsible for the frequency comb generation ${ }^{3}$.

\section{Temporal Evolution Model}

For silicon resonators, the usual Lugiato-Lefever equation ${ }^{2,3}$ should be extended to a generalized nonlinear envelope equation ${ }^{4}$ (GNEE), which describes in compact form the nonlinear ultrabroadband propagation of the pulse envelope in the presence of linear loss and dispersion, Kerr effect, Raman scattering, two-photon absorption (TPA) and free-carrier absorption (FCA):

$$
\begin{aligned}
& {\left[\partial_{\tau}-D+\frac{\alpha}{2}+i \delta_{0}+\frac{\sigma}{2}(1+i \mu) N_{c}\right] A(\tau, t)} \\
& =f_{0}+i \rho_{0}\left(1+i \tau_{s h} \partial / \partial t\right) p_{N L}^{(3)}(\tau, t, A)
\end{aligned}
$$

coupled with the carrier density $\left(N_{c}\right)$ equation

$$
\partial_{t} N_{c}=\frac{\beta_{T P A}}{2 \hbar \omega_{0}}|A|^{4}-\frac{N_{c}}{\tau_{c}} .
$$

Here $\tau$ is a slow time variable measuring successive circulations in the cavity, $t$ is a fast time for temporal field structures within the cavity, $D$ is the operator accounting for cavity dispersion, $\alpha$ and $\sigma$ are the linear loss and FCA coefficients, $\mu$ is the FCA induced phase-shift coefficient, $\rho_{0}=\omega_{0} / 2 n_{0} c \varepsilon_{0}, n_{0}=n\left(\omega_{0}\right), \omega_{0}$ is the pump carrier frequency, and $\beta_{\text {TPA }}$ is the TPA coefficient, $\delta_{0}$ is cavity detuning and $f_{0}$ is the injected cw pump amplitude. Moreover, the field is supposed to be periodic with the cavity circulation time $\tau_{R}, \quad A(\tau, t)=A\left(\tau, t+\tau_{R}\right)$. The nonlinear polarization reads as

$$
\begin{aligned}
& p_{N L}^{(3)}(\tau, t)=C_{3}\left[( 1 - \gamma _ { R } ) \left((A+i r \otimes A)|A|^{2}\right.\right. \\
& \left.+A^{3} \exp \left(-2 i \omega_{0} t\right) / 3\right) \\
& \left.+\gamma_{R} A \int_{-\infty}^{t} h_{R}\left(t-t^{\prime}\right)\left|A\left(t^{\prime}\right)\right|^{2} d t^{\prime}\right]
\end{aligned}
$$

where $C_{3}$ includes the frequency-dependent effective mode area, $\otimes$ denotes convolution product, $\gamma_{R}$ is the Raman fraction coefficient associated with the response function

$$
h_{R}=\left\{\begin{array}{c}
\exp \left(-t / \tau_{2}\right) \sin \left(t / \tau_{1}\right)\left[\left(\tau_{1}^{2}+\tau_{2}^{2}\right) / \tau_{1} \tau_{2}^{2}\right], t>0 \\
0 ; t<0
\end{array}\right.
$$


The frequency dependent TPA coefficient

$\hat{r}(\omega)=\beta_{T P A}(\omega) c / 2 \omega n_{2}$

is included according to the analytical expression for $\beta_{\mathrm{TPA}}(\omega)$ (see Fig. 1$)^{5}$, and $n_{2}=3 \chi^{(3)} / 8 n_{0}$.

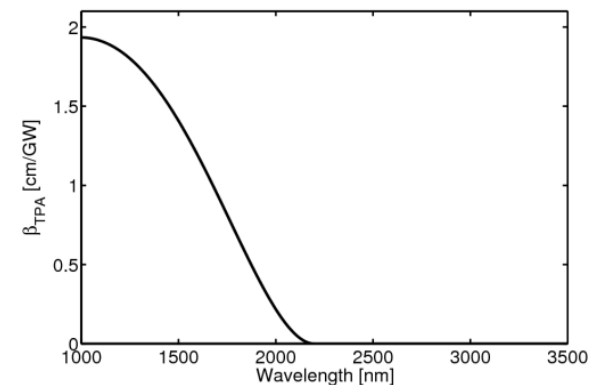

Fig. 1: Wavelength dependence of TPA coefficient.

\section{Analytical $\mu$-ring Design}

We tailor the linear dispersion and effective area properties of the silicon microrings as outlined in Fig. 2, for optimal MIR octave frequency comb generation by suitably designing the microresonator radius and cross section. In fact, the shape of the frequency combs can be controlled through the geometric contribution to the cavity mode effective refractive index. The eigenmode/eigenvalue problem is commonly numerically solved by using mode solvers, but we have been able to obtain analytical estimates for the spatial mode structure of silicon microresonators ${ }^{6}$. The analytical design has the merit of providing additional insight, and it is a very helpful tool for device optimization as it leads to rapid approximations. We derived a closed form analytical estimate for the propagation constant that is valid for resonators with a large radius to width ratio.

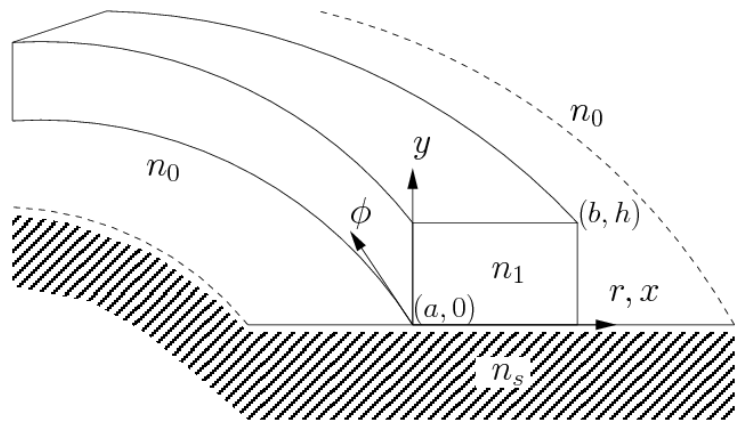

Fig. 2: Cross section showing the layer structure of the ring waveguide.

Fig. 3 illustrates examples of computed analytical dispersion and effective area profiles for TM and TE modes of a $50 \mu \mathrm{m}$ radius ring, with a $1 \mu \mathrm{m}$ wide $500 \mathrm{~nm}$ thick waveguide.
Comparison with full mode solver solutions confirms the validity of the analytical predictions in the $1.5 \mu \mathrm{m}-3.5 \mu \mathrm{m}$ range. Whereas in Fig. 4 we show the analytical dispersion profiles obtained with larger waveguide dimensions: as can be seen, the absolute value of dispersion is much reduced with respect to the case of Fig. 3 .

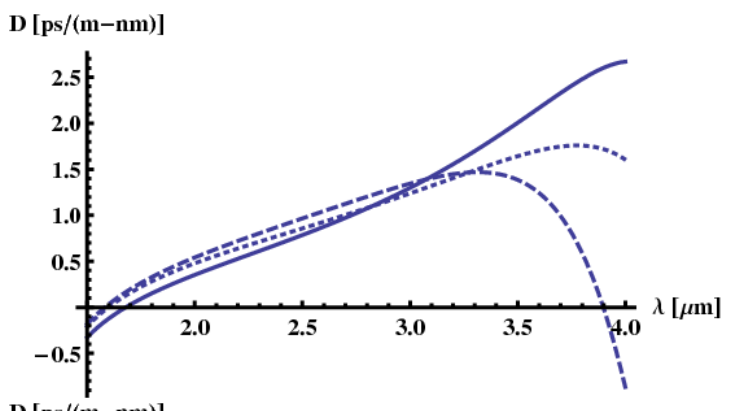

D [ps/(m-nm)]

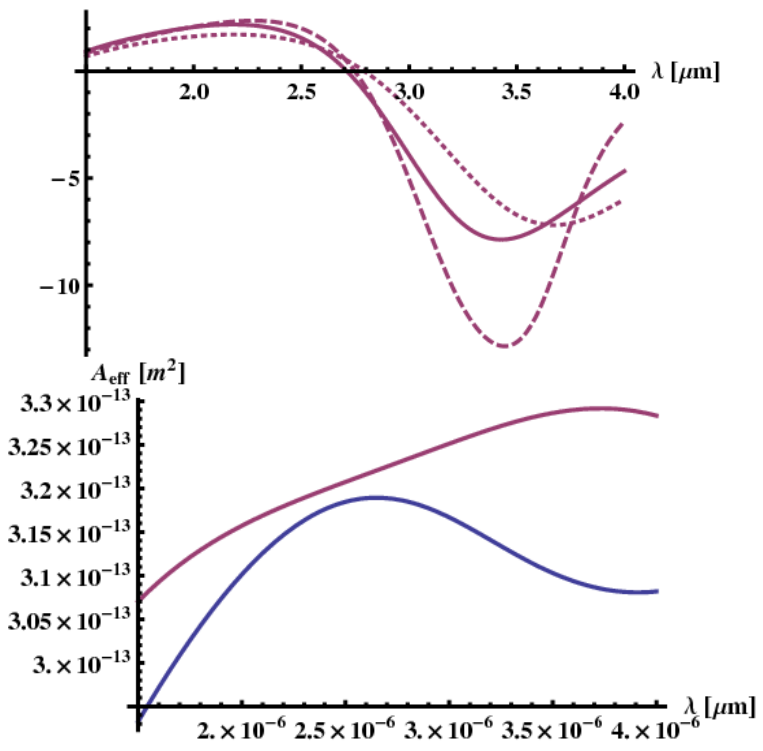

Fig. 3: Top: dispersion $D$ for the TM mode of a silicon ring resonator with $\mathrm{a}=49.5 \mu \mathrm{m}, \mathrm{b}=50.5 \mu \mathrm{m}$, and $\mathrm{h}=0.5 \mu \mathrm{m}$ (see Fig.1). Fully drawn lines: analytical; dotted lines: numerical from consistency requirements; dashed lines: numerical from mode solver.; Center: TE mode; Bottom: effective mode area wavelength dependence.

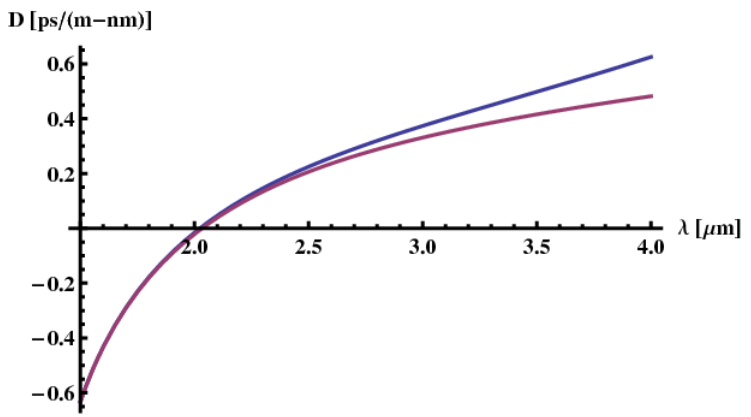

Fig. 4: Same as in Fig.3, for TM (blue) and TE (red) modes of a $\mu$-ring with waveguide dimensions of $1.5 \times 1.5 \mu \mathrm{m}$. 


\section{MIR Frequency Combs}

Based on the analytical design of the spatial mode properties of the $\mu$-rings, we numerically simulated the temporal dynamics of MIR frequency comb generation using Eqs.(1-3).
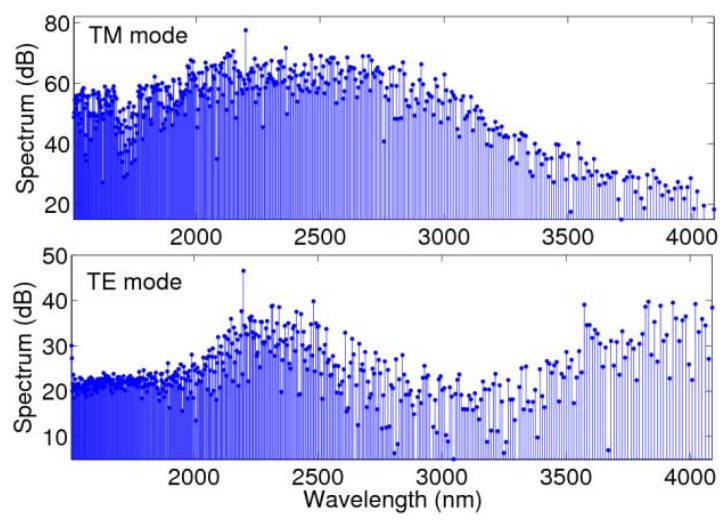

Fig. 5: Frequency comb generation from TM or TM modes of $\mu$-rings as in Fig. 3. The free-spectral-range at $2.2 \mu \mathrm{m}$ is 224 (246) GHz for the TE (TM) mode.

Fig. 5 compares the frequency combs obtained with a cw pump at $2.2 \mu \mathrm{m}$ coupled to either the TM or TE mode of the rings of Fig.3, respectively. Although a wider comb is obtained around the TM pump because of the uniform anomalous dispersion, the TE-pumped comb exhibits a considerable spectral broadening in the normal dispersion region 3.5-4 $\mu \mathrm{m}$ owing to dispersive wave emission.
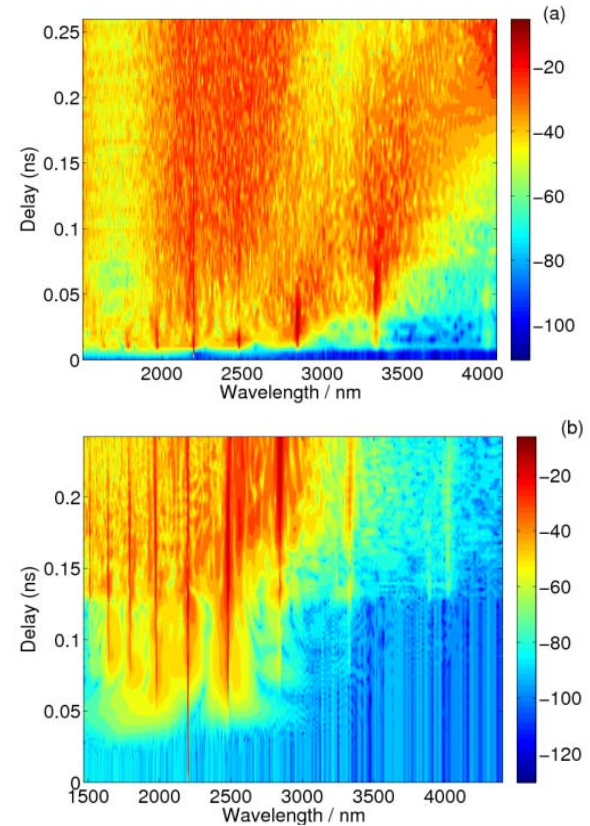

Fig. 6: Dynamics of frequency comb generation from TM modes of small $\mu$-rings as in Fig. 3 (a) or large $\mu$-ring as in Fig.4.
Fig. 6 compares the slow temporal dynamics of TM-pumped comb generation in either small waveguide rings (as in Fig. 3, see Fig. 6(a)) or larger waveguide rings (as in Fig. 4, see Fig. $6(\mathrm{~b}))$. As it can be seen, because of their smaller effective area, spectrally wider combs are obtained for the smaller rings, in spite of the relatively larger absolute value of the anomalous dispersion.

Additionally, we found that the presence of Raman scattering in silicon has a fundamental role in enhancing the emergence of a primary frequency comb based on modulation-instability activated, cascaded four-wave mixing as shown in Fig. 6(b).

\section{Conclusions}

Silicon $\mu$-ring resonators may enable a new class of chip-based, low pump power frequency comb sources in the MIR spectral region. We carried out a device optimization by means of an original and highly computationally efficient approach, involving the analytical design of the resonator spatial modes and the temporal study of ultra-wideband comb dynamics based on the GNEE approach.

\section{Acknowledgements}

We gratefully acknowledge support from Cariplo Foundation (grant no. 2011-0395), and the Italian Ministry of University and Research (MIUR, Project Nb. 2012BFNWZ2).

\section{References}

[1] P. Del'Haye, et al., "Optical frequency comb generation from a monolithic microresonator," Nature, Vol. 450, p. 1214 (2007).

[2] A. B. Matsko, A. A. Savchenkov, W. Liang, V. S. Ilchenko, D. Seidel, and L. Maleki, "Mode-locked Kerr frequency combs," Opt. Lett. Vol. 36, p.2845 (2011).

[3] T. Hansson, D. Modotto, and S. Wabnitz, "Dynamics of the modulational instability in microresonator frequency combs," Phys. Rev. A Vol. 88, p.023819 (2013).

[4] G. Genty, et al., "Nonlinear envelope equation modeling of sub-cycle dynamics and harmonic generation in nonlinear waveguides," Optics Express, Vol. 15, p. 5382 (2007).

[5] A. D. Bristow et a., "Two-photon absorption and Kerr coefficients of silicon for 850-2200 nm," Appl. Phys. Lett.Vol. 90, p. 191104 (2007).

[6] T. Hansson, D. Modotto, and S. Wabnitz, "Analytical approach to the design of microring resonators for nonlinear four-wave mixing applications," J. Opt. Soc. Am. B, Vol. 31, p. 1109 (2014) 